\title{
Improved ultrasonic sound sources utilizing circular diaphragms for generating convergent fields in air and their far-field properties
}

\author{
Kiichiro Matsuzawa, Takahi Hasegawa, and Masayuki Ochi \\ Department of Physics, Faculty of Science, Ehime University, \\ Bunkyo-cho, Matsuyama, 790 Japan
}

(Received 27 June 1980)

\begin{abstract}
This paper describes an improved model of the sound sources which were reported in the previous paper [K. Matsuzawa, Acustica 37, 241-248 (1977)]. Each sound source consists of a vibrator for use at $20 \mathrm{kHz}$, a metallic horn, and a thin metallic diaphragm in flexural vibration. The efficiency and some of the near-field properties for this improved model were reported in the previous short note [Y. Sasaki et al., J. Acoust. Soc. Jpn. (E) 1, 209-210 (1980)]. In this paper, the far-field properties are described and in addition near-field properties. The intense sound field near the diaphragm is confined in a region of some centimeters. The radiation pattern at $1 \mathrm{~m}$ distance has a single peak or double peaks about a peculiar direction. Numerical studies have been made using the theoretical formulas given in the previous paper. Though the improvement in strength caused a little discrepancy between the real construction and the theoretical counterpart, the experimental results are in good agreement with the calculated ones with respect to both the near-field and the far-field properties.
\end{abstract}

PACS number: 43. 88. Ar, 43. 35. Yb, 43. 25. Vt

\section{INTRODUCTION}

It has been known that effective radiation of ultrasonic power in air is brought about with a thin metallic diaphragm in resonant vibration. ${ }^{1-3)}$ Several years ago, one of the authors made sound sources which consisted of vibrators for use at 20 $\mathrm{kHz}$, metallic horns, and circular diaphragms vibrating in flexural modes of seven or eight nodal circles. $\left.{ }^{4}{ }^{5}\right) \mathrm{He}$ also found that their efficiency was about $80 \%$ and their sound pressure level with an electric power of $10 \mathrm{~W}$ was higher than $160 \mathrm{~dB}(0$ $\mathrm{dB}=20 \mu \mathrm{Pa}$ ) by the convergence effect. ${ }^{4,5)}$ But, their construction is not so strong; the maximum electric power which was able to be applied to them was 10 to $20 \mathrm{~W}$. Recently, improved sound sources having a rugged construction were made by Sasaki and the authors; ${ }^{6)}$ titanium alloy was used as the horn material instead of duralumin, the diameter of the horn top was increased from 6 to $10 \mathrm{~mm}$, and PZT vibrators were used instead of ferrite vibrators. The efficiency of the improved model was higher than $85 \%$, a sound pressure level of $170 \mathrm{~dB}$ was obtained with an electric power of $40 \mathrm{~W}$, and the possible electric power was up to $100 \mathrm{~W}{ }^{6)}$

As for the near fields of the old model, the experimental results have been found to be in good agreement with the theoretically calculated.5) The similar study for the improved model must be made because its horn-top diameter is increased, while in the theory it is assumed that the diaphragm is driven only at its center. In the present paper, this study is described together with some additional experiments.

Since the research was begun in order to produce an intense near field, the far-field property has not yet been examined. In the present paper, the experimental and calculated results for the sound 
pressure at $1 \mathrm{~m}$ distance are described. A decade ago, one of the authors examined the far-field radiation pattern of a rectangular diaphragm on which a one-dimensional flexural wave (wavelength $\lambda_{\mathrm{d}}$ ) was progressing. ${ }^{2)}$ He found that the angle between the normal line to the diaphragm and the direction of the peak of the pattern was $\sin ^{-1}\left(\lambda_{a}\right)$ $\lambda_{\mathrm{d}}$ ), where $\lambda_{\mathrm{a}}$ was the wavelength of sound in air. ${ }^{2)}$ Somewhat similar property is also found for the patterns in the circular diaphragms.

\section{SOUND SOURCES AND SOME EXPERIMENTS}

The photograph of one of the improved model is shown in Fig. 1. The details of the construction have already been reported in Ref. 6). Six sound sources were constructed by the combination of six diaphragms of different diameters and one vibratorhorn-nut system. The values of the diameter $2 a$ are listed in Table 1.

The experimental results described in Sec. 2, 3, and 4 were obtained by the same methods as explained in Ref. 5) and 6).

The resonant frequency and the efficiency were obtained by the admittance locus method, as listed in Table 1. The efficiency is very high as partly reported in Ref. 6). The $Q$ in air is of the order of $10^{3}$. Since the $Q$ is high even in air, it is reasonable to consider that the relative amplitude distribution over the diaphragm in air is almost the same as that in a vacuum, and also that the formulas for the amplitude distribution in Ref. 5) hold in the case of the diaphragm in air.

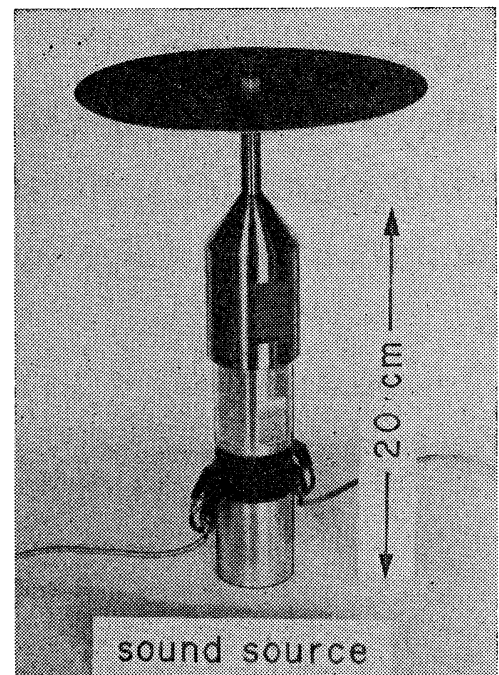

Fig. 1 Photograph of the sound source.

It has been found that $Y_{0}$ (the diameter of the motional admittance circle in air) as a function of $2 a$ has the maximum at $2 a=164 \mathrm{~mm}^{6}{ }^{6}$ To the sound source with this diaphragm, an electric power up to $100 \mathrm{~W}$ was safely applied. ${ }^{6)}$ But, to the other five sound sources, the application of $100 \mathrm{~W}$ was not always possible because an undesirable vibration occurred with a loud audible sound; the reason is not clear and is to be studied in future. In the present study, the experiments mentioned in Sec. 4 were made only on the diaphragm of $2 a=164 \mathrm{~mm}$, and the experiments in Sec. 6 were performed with an electric power of $10 \mathrm{~W}$. Additional experiments

Table 1 Some properties of sound sources and comparison between experimental and calculated values of the maximum sound pressure level at $1 \mathrm{~m}$ distance for an electric input power of $10 \mathrm{~W}$.

\begin{tabular}{|c|c|c|c|c|c|c|c|c|c|}
\hline \multicolumn{3}{|c|}{ Diameter of diaphragm $2 a(\mathrm{~mm})$} & 154.0 & 158.0 & 162.0 & 164.0 & 167.0 & \multicolumn{2}{|c|}{170.0} \\
\hline \multicolumn{3}{|c|}{ Resonant frequency $(\mathrm{kHz})$} & 20.67 & 20.21 & 20.02 & 19.93 & 19.79 & \multicolumn{2}{|c|}{19.63} \\
\hline \multicolumn{3}{|c|}{ Efficiency } & 0.85 & 0.86 & 0.84 & 0.86 & 0.87 & \multicolumn{2}{|c|}{0.89} \\
\hline \multicolumn{3}{|l|}{$2 a / \lambda_{\mathrm{d}}$} & 7.08 & 7.21 & 7.34 & 7.40 & 7.51 & \multicolumn{2}{|c|}{7.58} \\
\hline \multirow[t]{2}{*}{$\lambda_{\mathrm{d}} / \lambda_{\mathrm{a}}$} & & & 1.309 & 1.286 & 1.284 & 1.282 & 1.280 & \multicolumn{2}{|c|}{1.280} \\
\hline & & & & & & & & $\begin{array}{l}\text { Inner } \\
\text { peak }\end{array}$ & $\begin{array}{l}\text { Outer } \\
\text { peak }\end{array}$ \\
\hline \multirow{3}{*}{$\begin{array}{c}\text { Maximum sound } \\
\text { pressure level } \\
\text { (dB re } 20 \mu \mathrm{Pa}) \\
\text { at } 1 \mathrm{~m} \text { distance } \\
\text { for an electric } \\
\text { power of } 10 \mathrm{~W}\end{array}$} & \multicolumn{2}{|c|}{$\begin{array}{l}\text { Experiment } \\
\text { (without baffle) }\end{array}$} & 125.5 & 126.5 & 126.5 & 125.5 & 125.0 & 124.5 & 123.5 \\
\hline & & Rigorous & 127.2 & 126.8 & 126.2 & 125.9 & 124.9 & 124.1 & 125.2 \\
\hline & $\begin{array}{l}\text { Calcu- } \\
\text { lation }\end{array}$ & $\begin{array}{l}\text { Approx- } \\
\text { imate }\end{array}$ & 127.3 & 126.9 & 126.3 & 126.0 & 125.1 & 124.3 & 125.2 \\
\hline
\end{tabular}




\section{K. MATSUZAWA et al: : ULTRASONIC SOUND SOURCES IN AIR}

were made with other three vibrator-horn-nut systems of slightly different dimensions. For each system, $Y_{0}$ was examined for different $2 a$, and the diaphragm corresponding to the maximum $Y_{0}$ was selected. As far as this diaphragm was used for each system, the application of electric power up to $100 \mathrm{~W}$ was always possible.

\section{DIAMETER OF NODAL CIRCLES}

The nodal patterns on the six diaphragms were examined. In each case, seven concentric nodal circles were observed clearly. The diameter $2 r_{N}$ of nodal circle was measured, and some examples are listed in the "Exp." columns in Table 2. The bestfitted values of $2 a / \lambda_{\mathrm{d}}$ and the calculated values of $2 r_{N}$ were determined on the basis of the procedure explained in Ref. 5). They are listed in Table 1 and in the "Calc." columns in Table 2.

It has been known that $2 a / \lambda_{\mathrm{d}}$ is 6.99 for an unconstrained diaphragm with seven nodal circles, 7.49 for a center-clamped one with seven nodal circles, and 7.99 for the unconstrained with eight nodal circles. ${ }^{7)}$ In Table $1,2 a / \lambda_{\mathrm{d}}$ varies from 7.08 to 7.58. Therefore, corresponding to the variation of the diameter $2 a$ from 154 to $170 \mathrm{~mm}$, the modal pattern varies from what is almost the same as the unconstrained diaphragm with seven nodal circles, by way of the pattern of seven nodal circles for center-clamped diaphragm, to that of a centerloaded diaphragm with eight nodal circles. But the smallest nodal circle in the last cases was not observed because of the larger horn top of $10 \mathrm{~mm}$ diameter.

As seen in Table 2, the experimentally obtained

Table 2 Comparison between experimental and calculated diameters of nodal circles on the diaphragms.

\begin{tabular}{|c|c|c|c|c|}
\hline \multirow{2}{*}{$\begin{array}{l}\text { Diameter of } \\
\text { diaphragm } 2 a \\
(\mathrm{~mm})\end{array}$} & \multicolumn{2}{|c|}{154.0} & \multicolumn{2}{|c|}{170.0} \\
\hline & $\begin{array}{l}\text { Exp. } \\
17.0\end{array}$ & $\begin{array}{r}\text { Calc. } \\
18.6\end{array}$ & Exp. & Calc. \\
\hline \multirow{7}{*}{$\begin{array}{l}\text { Diameter of } \\
\text { nodal circle } \\
2 r_{N}(\mathrm{~mm})\end{array}$} & 39.9 & 40.1 & 30.6 & 30.3 \\
\hline & 61.9 & 61.8 & 52.7 & 52.5 \\
\hline & 83.5 & 83.5 & 74.9 & 74.9 \\
\hline & 105.2 & 105.3 & 97.2 & 97.3 \\
\hline & 126.9 & 127.1 & 119.8 & 119.7 \\
\hline & 147.1 & 146.9 & 142.3 & 142.3 \\
\hline & & & 162.4 & 162.7 \\
\hline
\end{tabular}

$2 r_{N}$ is in good agreement with the calculated $2 r_{N}$, except for the inner nodal circles. On the contrary, in Table II of Ref. 5), the agreement between the two kinds of the $2 r_{N}$ values is good even at the inner nodal circles. This difference is inferred to be caused by the difference in the top diameter of the horn.

As listed in Table 1, the values of $\lambda_{\mathrm{d}} / \lambda_{\mathrm{a}}$ are obtained from the values of $2 a, 2 a / \lambda_{\mathrm{d}}$, and the resonant frequency in Table 1 and the sound velocity in air at the room temperature. These values are used in the calculations mentioned in Sec. 4 and 6.

\section{INTENSE SOUND FIELD NEAR THE DIAPHRAGM}

As shown by the dashed curves in Figs. 2(a) and 2(b), the relative sound pressure levels were measured along the central axis of the sound source and along a line which crossed the central axis at the right angle at the point of the maximum sound pressure, respectively. The solid curves show the calculated results obtained by the same way as those in Figs. 10(b) and 10(c) of Ref. 5). In Fig. 2(a), the two

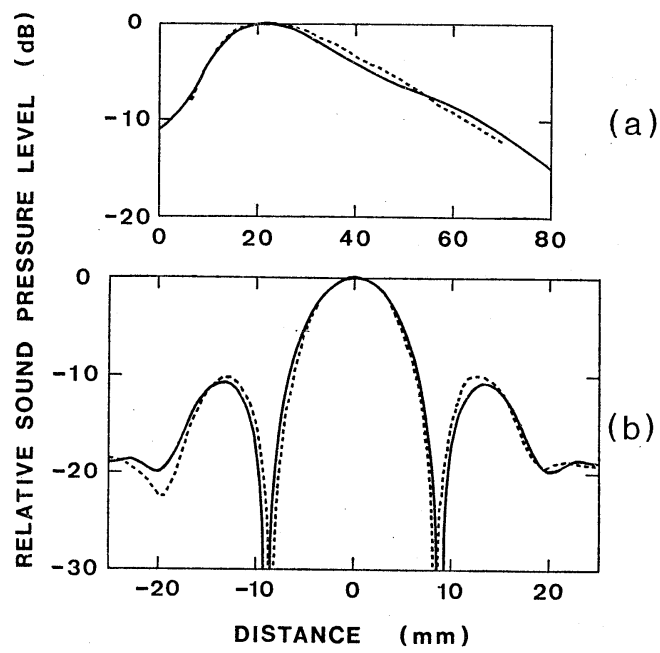

Fig. 2 Comparison of calculated sound pressure (solid curves) with experimental data (dashed curves) for the sound source with the diaphragm of $164 \mathrm{~mm}$ in diameter.

(a) Relative level on the central axis versus distance from the diaphragm,

(b) relative level on the line which crosses the central axis at the right angle at the point of $20 \mathrm{~mm}$ from the diaphragm versus distance from the central axis. 
curves are in good agreement with each other. On the other hand, the agreement in Fig. 2(b) is somewhat poor. Corresponding results for the old model are seen in Fig. 10(c) of Ref. 5), where the agreement is very good. This difference is presumed to be caused by the difference in the top diameter of the horn. But in summary, the local convergence effect is the same for the two models.

The electric power for producing a sound pressure level of $170 \mathrm{~dB}$ at the highest point in Fig. 2 is calculated in the following way; (i) the value of $\xi$ (rms amplitude of diaphragm edge) is obtained from the value of the measured sound pressure $|p|$ and Eq. (10) in Ref. 5), (ii) the total sound power is computed from Eq. (11) in Ref. 5) and the value of $\xi$, and (iii) the electric power is determined from the measured efficiency in Table 1 and the total sound power. Thus, the calculated electric power is $37 \mathrm{~W}$ and is found to be in good agreement with the experimental value, $40 \mathrm{~W}$.

\section{EXPERIMENTAL ARRANGEMENT AND THEORETICAL PREPARATION FOR THE SOUND PRESSURE AT $1 \mathrm{~m}$ DISTANCE}

The arrangement of a sound source and a baffle is shown in Fig. 3. The baffle is made of an aluminum plate ( $1 \mathrm{~mm}$ thick) of 30 to $40 \mathrm{~cm}$ in size. The shape of the outer periphery is somewhat irregular. The diameter of the circular opening of the baffle is made $3 \mathrm{~mm}$ larger than the diameter of the

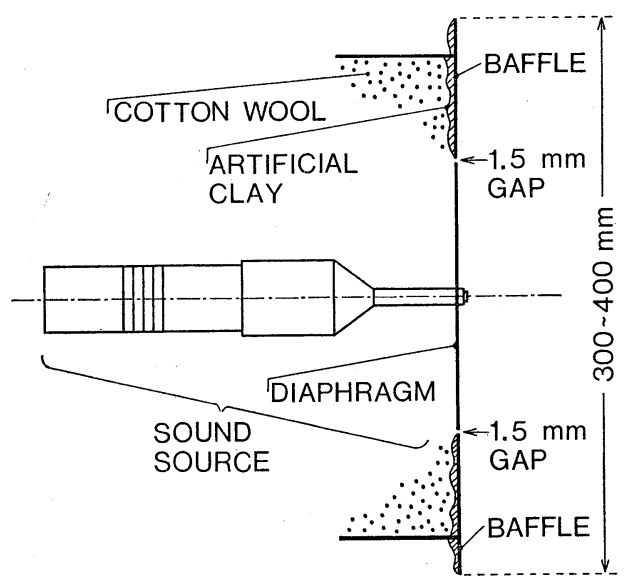

Fig. 3 Arrangement of the sound source and the baffle when the sound pressure at $1 \mathrm{~m}$ distance is measured. diaphragm. Therefore, the gap between the diaphragm and the baffle is $1.5 \mathrm{~mm}$ in average. If the gap was too small, the baffle would happen to touch the diaphragm and the vibration ceased. The rear side of the baffle is covered with artificial clay. Cotton wool over it acts as a sound absorbing material.

The sound source was put on a plastic foam plate, which was not shown in Fig. 3. The vibrator and the horn were on the foam plate, so that the central axis was horizontal. Bars which were tightly fixed to the baffle were tied with cords to posts that also supported the foam plate. Accordingly, the baffle could easily be attached to, or detached from, the sound source. The posts were fixed to an immovable stand. A microphone (Brüel and Kjaer, Type 4135 or Type 4136) was fixed to an arm, which was attached to the stand so as to rotate around it in a horizontal plane. The distance between the microphone and the diaphragm center of the sound source was $1 \mathrm{~m}$. A distance of more than $1 \mathrm{~m}$ was difficult to be achieved because the size of the anechoic room was not large enough.

Let $\varphi$ denote the angle between the central axis of a sound source and the line from the diaphragm center to a measurement point. When the measurement point is far enough, Eq. (11) in Ref. 5) holds. The rigorous sound pressure at $1 \mathrm{~m}$ distance is computed as a function of $\varphi$ by using Eq. (10) in Ref. 5), where the double integration is made numerically. On the other hand, the approximation of the far-field sound pressure is given by

$$
|p|=\omega^{2} \rho \xi \frac{a^{2}}{R_{0}}|\Psi|,
$$

where $R_{0}$ is the distance of $1 \mathrm{~m}$ and other quantities have been defined in Ref. 5). The magnitude of $\Psi$ which is a function of $\varphi$ gives the relative radiation pattern at the infinite distance.

\section{SOUND PRESSURE AT $1 \mathrm{~m}$ DISTANCE}

\subsection{Radiation Patterns}

The radiation patterns of the sound sources were measured with and without the baffles. An example is shown in Fig. 4(a). No systematic difference is found for these two patterns. Thus, the effect of the baffle is negligible.

The patterns at $1 \mathrm{~m}$ distance and those at the infinite distance are calculated from Eq. (10) in Ref. 

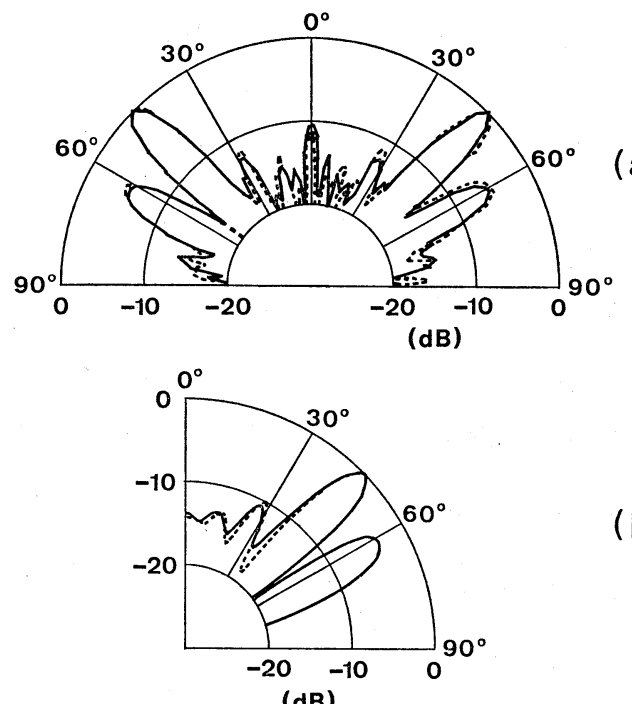

Fig. 4 Radiation patterns of the sound source with the diaphragm of $164 \mathrm{~mm}$ in diameter.

(a) Comparison between measured patterns at $1 \mathrm{~m}$ when the baffle is used (dashed curve) and when no baffle is used (solid curve),

(b) comparison between calculated patterns for $1 \mathrm{~m}$ distance (solid curve) and for the infinite distance (dashed curve).

5) and Eq. (1), respectively. As shown in Fig. 4(b), the two patterns are in good agreement, especially it is so for the main lobe. It may be considered that $1 \mathrm{~m}$ is close enough to the infinite distance.

From Fig. 4, it is found (i) that there is some small but clear discrepancy between the pattern measured without the baffle and the calculated pattern at $1 \mathrm{~m}$ distance, and (ii) that this discrepancy is not narrowed if the baffle is provided in the experiment. Therefore, it may be reasonable to conclude that the discrepancy would hardly disappear if the gap between the baffle and the diaphragm could be removed in the experiment, and that the baffle is not a main cause of the discrepancy.

Figure 5 shows the calculated and measured patterns at $1 \mathrm{~m}$ when the baffles are used. The two curves in each pair are in good agreement as for their main lobes, but small discrepancies are found except for these main lobes. One of the causes of the discrepancies may be the finite size of the diameter of the horn top.

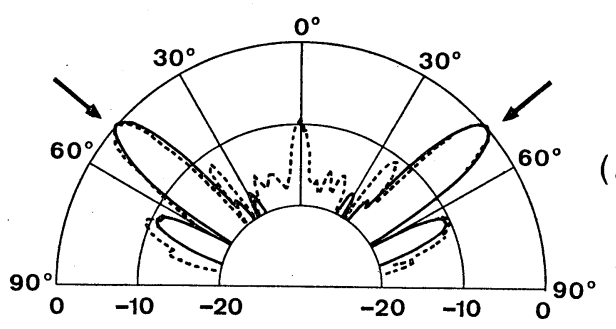

(a)

(dB)

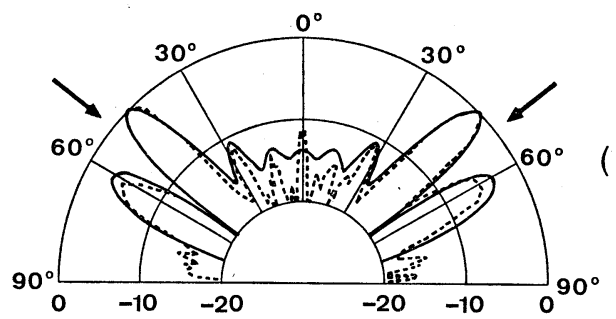

(dB)

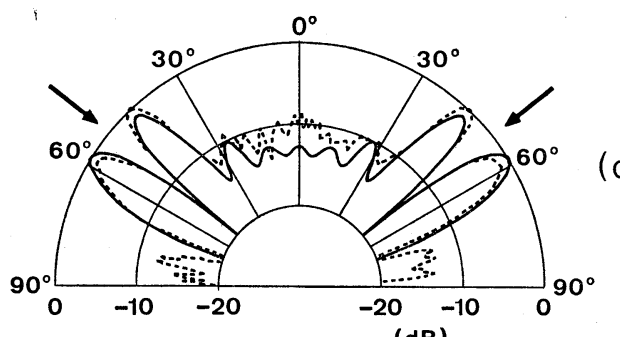

(dB)

Fig. 5 Comparison between calculated (solid curves) and measured (dashed curves) radiation patterns at $1 \mathrm{~m}$ for the sound sources with the baffles.

Arrow marks indicate the angles $\sin ^{-1}\left(\lambda_{\mathrm{a}}\right)$ $\lambda_{\mathrm{d}}$ ). The diameters of the diaphragms are (a) $154 \mathrm{~mm}$, (b) $164 \mathrm{~mm}$, and (c) $170 \mathrm{~mm}$.

The arrow marks in Fig. 5 show the angles of $\varphi_{0}=\sin ^{-1}\left(\lambda_{\mathrm{a}} / \lambda_{\mathrm{d}}\right)$. The main lobes in Fig. 5 (a) have sharp single peaks at $\varphi_{0}$, while those in Fig. 5(c) have sharp double peaks with deep dips at $\varphi_{0}$. The main lobes in Fig. 5(b) are of the intermediate shape between (a) and (c). This special character of the main lobes is ascertained both in experiments and calculations. The factor which determines this character will be studied in future.

\subsection{Maximum Sound Pressure Level}

The maximum sound pressure level at $1 \mathrm{~m}$ distance was measured for every sound source without the baffle for an electric power of $10 \mathrm{~W}$. The reproducibility of the measurements was within the 
range of $\pm 0.5 \mathrm{~dB}$. As shown in the third row from the bottom of Table 1, the maximum level is about $125 \mathrm{~dB}(36 \mathrm{~Pa})$. For the diaphragm of $2 a=170 \mathrm{~mm}$, both double peaks were examined.

The maximum sound pressure level difference was examined for the cases with and without the baffle. In most cases, the level of the cases with the baffle is lower. It is found that the difference is $0.0 \sim 0.3 \mathrm{~dB}$. Since the difference is somewhat smaller than the range of reproducibility $( \pm 0.5 \mathrm{~dB})$, the effect of the baffles is reasonably neglected. The effect of the gap between the baffle and the diaphragm would be negligible also.

The maximum level is calculated in the reverse process that is described in the last part of Sec. 4. The rigorous values from Eq. (10) of Ref. 5) and the approximate values from Eq. (1) are listed in the second row from the bottom and the lowest row of Table 1, respectively. Since the difference between these calculated values is $0.0 \sim 0.2 \mathrm{~dB}$, the distance of $1 \mathrm{~m}$ may be considered to be almost infinite.

As seen in the third and second rows from the bottom of Table 1, the agreement between the measured and calculated values is very good except for the two cases at the both ends of the table, these are also fairly good.

\section{CONCLUSION}

The six sound sources of the improved model have the following distinctive properties: (i) the efficiency is high, (ii) as for the radiation pattern at $1 \mathrm{~m}$ distance, the single peak or the double peaks appear in the direction which lies at the angle of $\sin ^{-1}\left(\lambda_{\mathrm{a}} / \lambda_{\mathrm{d}}\right)$ to the central axis, and (iii) the sound pressure level at a distance of $1 \mathrm{~m}$ to the maximum pressure direction is about $125 \mathrm{~dB}$ for an electric power of $10 \mathrm{~W}$.

The measured sound pressure levels at $1 \mathrm{~m}$ distance were almost independent of the presence of the baffles. The rigorously calculated sound pres- sure levels at $1 \mathrm{~m}$ distance are almost equal to the levels obtained from the approximate formula for the infinite distance.

For one sound source selected from the six, the following two points are ascertained with high electric power applied: (i) the improvement in strength and (ii) the convergence in the near field.

In spite of the increased horn-top diameter of 10 $\mathrm{mm}$, the experimental results are in good agreement with the calculated ones on the whole.

In the near future, a theoretical study with respect to the peculiar property of the far-field radiation patterns will be made.

\section{REFERENCES}

1) M. Kawamura, H. Yamane, T. Iimori, and K. Seya, "Aerial intense ultrasound generator consisting of higher mode flexural vibrating plate, metallic horn and magnetostrictive vibrator, and its applications," J. Acoust. Soc. Jpn. 23, 134 145 (1967) (in Japanese).

2) K. Matsuzawa, "Ultrasonic transducers with flexurally vibrating diaphragms for use in air, I and II,” Jpn. J. Appl. Phys. 9, 235-245 and 11671171 (1970).

3) J. A. G. Juárez and G. R. Corral, "Piezoelectric transducer for air-borne ultrasound," Acustica 29, 234-239 (1973).

4) K. Matsuzawa, "Sound sources for producing intense ultrasonic fields in small regions in air," Proc. 8th Int. Congr. Acoust., London 2, 433 (1974).

5) K. Matsuzawa, "Sound sources utilizing flexurally vibrating circular diaphragms for generating convergent ultrasounds in air," Acustica 37, 241-248 (1977).

6) Y. Sasaki, K. Matsuzawa, M. Ochi, and T. Hasegawa, "High efficiency ultrasonic sound source in air," J. Acoust. Soc. Jpn. (E) 1, 209-210 (1980).

7) A. W. Leissa, Vibration of Plates (NASA, Washington, D. C., 1969), pp. 10-11 and 17-19. 